\title{
BMJ Open Problem and non-problem gamblers: a cross-sectional clustering study by gambling characteristics
}

\author{
Morgane Guillou Landreat (1) , 1,2,3 Isabelle Chereau Boudet, ${ }^{4}$ Bastien Perrot, ${ }^{3}$ \\ Lucia Romo, ${ }^{5,6}$ Irene Codina, ${ }^{7}$ David Magalon, ${ }^{8}$ Melina Fatseas, ${ }^{9,10}$ \\ Amandine Luquiens (D) , ${ }^{11,12,13}$ Georges Brousse, ${ }^{14}$ Gaëlle Challet-Bouju, ${ }^{15}$ \\ Marie Grall-Bronnec, ${ }^{16}$ JEU-Group
}

To cite: Guillou Landreat M, Chereau Boudet I, Perrot B, et al. Problem and non-problem gamblers: a cross-sectional clustering study by gambling characteristics. BMJ Open 2020;10:e030424. doi:10.1136/ bmjopen-2019-030424

- Prepublication history for this paper is available online. To view these files, please visit the journal online (http://dx.doi. org/10.1136/bmjopen-2019030424).

Received 22 March 2019 Revised 25 November 2019 Accepted 05 December 2019

Check for updates

(C) Author(s) (or their employer(s)) 2020. Re-use permitted under CC BY-NC. No commercial re-use. See rights and permissions. Published by BMJ.

For numbered affiliations see end of article.

Correspondence to

Professor Marie Grall-Bronnec marie.bronnec@chu-nantes.fr

\section{ABSTRACT}

Objectives Gambling characteristics are factors that could influence problem gambling development. The aim of this study was to identify a typology of gamblers to frame risky behaviour based on gambling characteristics (age of initiation/of problem gambling, type of gambling: pure chance/chance with pseudoskills/chance with elements of skill, gambling online/offline, amount wagered monthly) and to investigate clinical factors associated with these different profiles in a large representative sample of gamblers.

Design and setting The study is a cross-sectional analysis to the baseline data of the french JEU cohort study (study protocol : Challet-Bouju et al, 2014). Recruitment (April 2009 to September 2011) involved clinicians and researchers from seven institutions that offer care for or conduct research on problemgamblers (PG). Participants were recruited in gambling places, and in care centres. Only participants who reported gambling in the previous year between 18 and 65 years old were included.

Participants gave their written informed consent, it was approved by the French Research Ethics Committee.

Participants The participants were 628 gamblers : 256 non-problem gamblers (NPG), 169 problem gamblers without treatment (PGWT) and 203 problem gamblers seeking treatment (PGST).

Results Six clustering models were tested, the one with three clusters displayed a lower classification error rate $(7.92 \%)$ and was better suited to clinical interpretation : 'Early Onset and Short Course' (47.5\%), 'Early Onset and Long Course' (35\%) and 'Late Onset and Short Course' (17.5\%). Gambling characteristics differed significantly between the three clusters.

Conclusions We defined clusters through the analysis of gambling variables, easy to identify, by psychiatrists or by physicians in primary care. Simple screening concerning these gambling characteristics could be constructed to prevent and to help PG identification. It is important to consider gambling characteristics : policy measures targeting gambling characteristics may reduce the risk of PG or minimise harm from gambling.

Trial registration number NCT01207674 (ClinicalTrials. gov); Results.
Strengths and limitations of this study

- The sample size of gamblers $(n=628)$ has rarely been achieved for studies with semi-structured interviews.

- The mixed sample of non-problemgamblers and problemgamblers who have or have not sought treatment is one of the main strengths of our study, with inclusion directly from gambling locations.

- Recruitment of patients was performed at the moment of the legalisation of online gambling in France, which limits generalisation, as online gambling has since become widely available.

- Risk factors are self-reported at baseline evaluation. In future studies, these results should be compared with longitudinal data.

\section{INTRODUCTION}

Gambling is a widespread social activity worldwide. Almost all national surveys conclude that there are more gamblers than nongamblers. ${ }^{1}$ For example, $74 \%$ of the general population in France had gambled in their lifetime. ${ }^{2}$ The gambling industry has developed many types of gambling on different media, especially on the Internet. This expansion of legalised gambling has been identified as a public health concern. ${ }^{3-5}$ Participation in gambling increases with gambling deregulation, prolific advertising and the growing availability of gambling. ${ }^{67}$

Social gambling may become a gambling disorder (GD) in a minority of cases. ${ }^{78}$ GD is defined as a persistent, maladaptive pattern of gambling resulting in clinically significant impairment or distress, according to the Fifth Edition of the Diagnostic and Statistical Manual of Mental Disorders. ${ }^{9}$ Around the world, between $0.2 \%$ and $2.1 \%$ of the population develops a GD, ${ }^{10}$ but a larger proportion experiences 'problem gambling', meaning having difficulties with gambling, but not as 
severe as those classified as GD. ${ }^{10}$ Throughout this paper, 'problem gamblers' or 'problem gambling' will refer to a heterogeneous group of persons or conditions encompassing GD and its subthreshold symptoms. The lifetime prevalence of problem gambling across the world ranges from $0.7 \%$ to $6.5 \%$. $^{1}$ In France, the last national survey estimated that $2.7 \%$ of the population could be considered problem gamblers. $^{2}$

The status of the problem gambler is unstable over time, ${ }^{11}$ and gamblers can have very different types of problem gambling development. Different factorsindividual, environmental or those linked to gambling behaviour characteristics-may influence gambling status and the development of problem gambling. ${ }^{1}$

Most of the studies of problem gambling risk factors have focused on individual or environmental factors. Problem gambling is more likely to occur among men, ${ }^{1} 2 \quad 11 \quad 12$ individuals with psychiatric disorders (anxiety, depression and attention deficit/hyperactivity disorder (ADHD)), ${ }^{11}$ or individuals of extreme old or young age. ${ }^{13-16}$ Environmental factors such as level of income, socioeconomic integration or social support have an influence both on problem gambling prevalence worldwide and on individual gambling development and transitions between social gambling and problem gambling. ${ }^{1811}$ Different clusters of problem gamblers emerged through the analysis of these clinical variables. ${ }^{17-21}$ Blaszczynski and Nower defined a theoretical pathway model of problem gambling. ${ }^{17}$ They identified three clusters of problem gamblers (conditioned gamblers, emotionally vulnerable gamblers and antisocial impulsivist gamblers) based mainly on clinical experience with problem gamblers and through analysis of psychopathological variables. They assumed that certain clinical variables, such as psychiatric comorbidities or impulsivity, could influence the development of problem gambling. Several empirical studies of this pathways model and other subtyping approaches confirmed clinical differences between problem gamblers' profiles, even though no study has conclusively supported this clinical classification and the underlying prognosis hypotheses. ${ }^{18-21}$ In all these studies, clustering was made through analysis of psychopathological variables measured with psychometrics tools and did not include gambling characteristics.

Only a few studies have analysed gambling characteristics and their influence on gambling status (social gambling or problem gambling) and problem gambling development. ${ }^{72}$ 21-26 Gambling characteristics include many variables: age of initiation, medium or type of gambling, gambling history, wagering amounts, frequency of gambling, gambling status (problem or non-problem gamblers), etc. An early age of initiation is a high risk factor for the development of problem gambling later in life and is associated with a higher level of problem gambling severity. ${ }^{21-23}$ Online gambling contributes to and enhances the risk of problem gambling development, ${ }^{6}{ }^{12}$ even if most online gamblers also gamble in offline forms. ${ }^{12}{ }^{24}$ When considering preferred gambling activity, gamblers who preferentially play the same type of games share a common profile independent of the pathological characteristics. ${ }^{25}$ Hing et al compared the profiles of online gamblers according to their preferred type of gambling (electronic gaming machine (EGM), horse racing or sport betting); they showed that online bettors were younger men, with more frequent substance use. ${ }^{7}$

All these previous studies were, in a large majority of cases, undertaken within the problem gambling population. ${ }^{19-21}$ 27-29 They analysed one specific gambling characteristic or defined clustering according to psychological variables and psychometric or cognitive assessment tests. $192729-31$

Combined with individual at-risk factors as psychopathological variables, clinicians should be aware of gambling characteristics.

We hypothesised that gambling structural characteristics influenced gamblers' profiles and problem gambling development, that clustering through gambling characteristics would help to identify different types of problem gamblers and that this classification would be clinically pertinent.

We did not find any studies in the literature which involved a broad sample of gamblers, included nonproblem gamblers and problem gamblers and specifically concerned gambling characteristics.

Our objective was to identify a typology of gamblers based on clinical and gambling characteristics, and to investigate factors associated with these different profiles in a large representative sample of gamblers.

The findings can help all clinicians, even non-specialists, to be aware of and identify problem gamblers through the evaluation of gambling characteristics. It may also improve gamblers interventions tailored to the specific gambling characteristics.

\section{MATERIAL AND METHODS \\ Participants}

The participants were 628 gamblers who took part in the JEU cohort study. The sample included 256 nonproblem gamblers (NPG), 169 problem gamblers without treatment (PGWT) and 203 problem gamblers seeking treatment (PGST). The JEU cohort study is a 5-year longitudinal national case-control cohort in France that seeks to identify protective and risk factors in gambling practice (for more information, please refer to the study protocol of the JEU cohort Challet-Bouju et al, 2014 ${ }^{32}$ ). The present study applied a cross-sectional analysis to the baseline data of the JEU cohort.

Recruitment occurred between April 2009 and September 2011 and involved a group of French clinicians and researchers from seven institutions that offer care for or conduct research on problem gambling.

The sample was constituted based on an approximate equality of size between problem gamblers and 


\begin{tabular}{|c|c|}
\hline Inclusion criteria & Exclusion criteria \\
\hline $\begin{array}{l}\text { Participants who reported } \\
\text { gambling on at least one } \\
\text { occasion in the previous year }\end{array}$ & No gambling in the past year \\
\hline Between 18 and 65 years old & $\begin{array}{l}\text { Under } 18 \text { or over } 65 \text { years old } \\
\text { cognitive impairment }\end{array}$ \\
\hline $\begin{array}{l}\text { Understanding of French } \\
\text { language }\end{array}$ & $\begin{array}{l}\text { Inability to understand the } \\
\text { French language }\end{array}$ \\
\hline Written consent & No consent \\
\hline
\end{tabular}

non-problem gamblers because of the low prevalence of gambling problems in the general population. Participants were recruited in different gambling places (casinos, cafés, smoke shops, etc) and via the press in order to cover the broadest possible range of gambling activities. In gambling places, all the gamblers were solicited outside of gambling time, because gamblers are particularly irritable when gambling and in order to avoid disturbing the gambling venue activity. We have no information about non-responders as a consequence of the arrangements with the gambling places which agreed to help us, whereby we sought no information from gamblers who refused to participate in the first instance. Problem gamblers were also recruited in seven care centres, where they had started treatment less than 6 months before. Only participants who reported gambling on at least one occasion in the previous year and who were between 18 and 65 years old were included in the study. Participants were given a compensation of $€ 15$, except for problem gamblers from care centres with whom the interview would have been held for care purposes anyway.

Inclusion and exclusion criteria are presented in table 1.

Participants were informed about the research and gave their written informed consent prior to their inclusion in the study.

\section{Patient and public involvement}

No patients nor the public were involved in the development of the research question. No patient advisers were included in the research project.

\section{Assessment}

The baseline assessment was performed just after inclusion in the study. The assessment combines a clinical structured interview carried out with a trained researcher or psychologist with a set of standardised self-report questionnaires. Participants completed the baseline interview in the research centre or the gambling place in which they were recruited.

\section{Sociodemographic characteristics}

We collected information about age, gender, marital status, income and work status.
Gambling characteristics

Pathological gambling section in the DSM-IV TR (APA 1994)

We used a clinical interview based on the 10 diagnostic criteria for pathological gambling according to the Diagnosticand Statistical Manual of Mental Disorders, Fourth Edition, Text Revision (DSM-IV TR) (AmericanPsychiatric Association (APA), 2000). The gambling disorder section of the DSM-5 could not have been used because the recruitment was conducted in 2009 to 2011. Gamblers who met at least three DSM-IV criteria were classified as problem gamblers, including both gamblers 'at risk' of pathological gambling and gamblers with a diagnosis of pathological gambling. We used a non-standard threshold of three instead of five so as to include subclinical forms of problem gambling. Previous literature has supported the relevance of this categorisation. ${ }^{33-35}$ The number of positive DSM-IV criteria for problem gambling was also used as a dimensional score of problemgamblers (PG) severity.

\section{Problem gambling development and characteristics}

We constructed a questionnaire to collect information concerning the course and characteristics of their gambling. The development of the gambling was approached by investigating the history of their gambling practice: age at initiation and of first gambling-related problems, duration of gambling history (from age at initiation to current age) and age at the time of the diagnosis of comorbid psychiatric disorders (if relevant). We collected information about the familial and social support attitudes towards the problem gambler's difficulties. The individual's experimentation with a gamblingfree period of at least 1 month's duration since his/her initiation was also investigated.

We collected information on participation in and frequency of various forms of gambling over the past year. This included the mean average amount of money wagered monthly in gambling and the maximum wager placed in a single day. With regard to gambling preferences, we investigated the preferred type of gambling and medium for gambling, presented in table 2. Gamblers identified their preferred gambling activity among all those they had experienced and we classified the preferred type of gambling according to the three categories proposed by Boutin in 2010: pure chance games (lotteries, slot machines, scratch cards, video lottery terminals, etc), bank games with an element of skill (sports betting, horse race betting, blackjack) or social games with skill (mainly Texas hold 'em and Omaha variants of poker). ${ }^{36}$ We also asked them to specify if they preferred gambling on the Internet or offline.

\section{Psychiatric comorbidities}

Mini-International Neuropsychiatric Interview

The Mini-InternationalNeuropsychiatric Interview is a structured diagnostic interview that is compatible with the International Statistical Classification of Diseases and Related Health Problems, Tenth Revision (ICD-10), and 
Table 2 Types and media of gambling and gambling status

Type of gambling

Pure chance games (video lottery terminals, scratch games, lotteries) Chance games with pseudoskills (sports betting, horse race betting, blackjack)

Chance games with element of skills (poker)

\begin{tabular}{ll} 
Medium of gambling & $\begin{array}{l}\text { Online: gambling on the Internet } \\
\text { Offline: smoke shop, café, casino, } \\
\text { etc }\end{array}$ \\
Gambling status & $\begin{array}{l}\text { Non-problem gambler } \\
\text { Problem gambler seeking treatment } \\
\text { Problem gambler without treatment }\end{array}$ \\
\hline
\end{tabular}

the Diagnostic and Statistical Manual of Mental Disorders, Fourth Edition (DSM-IV). ${ }^{37}$

It explores the lifetime and current main Axis I disorders (mood and anxiety disorders, psychotic syndrome, alcohol and substance use disorders).

\section{Wender Utah Rating Scale-Child}

This self-report questionnaire has been validated for the retrospective evaluation of childhood ADHD in adults. Its specificity (89.1\%) is good. It is designed to assess ADHD symptoms represented by 25 items on a 5 -point Likert scale. A score greater than or equal to $46 / 100$ would indicate that diagnosis. ${ }^{38} 39$

\section{Personality}

\section{A 125-item version of the temperament and character inventory}

The temperamentand character inventory, 125-item version, is used to rapidly explore the four dimensions of temperament and the three dimensions of character in personality as defined by Cloninger's psychobiological model. ${ }^{40}$ It measures seven dimensions through four temperaments and three characters. ${ }^{41}{ }^{42}$ The dimensions related to temperament (genetic and stable tendencies of personality) and the dimensions related to character (acquired under the influence of apprenticeship, experience and environment) were assessed.

\section{Statistical analysis}

A descriptive analysis of the sociodemographic, clinical and gambling characteristics was carried out in order to obtain means, medians and SD of continuous variables, as well as the number of people and the percentages of categorical variables.

To identify a typology of gamblers based on the course of their gambling and their preferences, we led an exploratory analysis. We performed a clustering of gamblers with eight variables: age at initiation, age at onset of gambling problem, duration of gambling history, age at diagnosis of comorbid psychiatric disorder, history of a gambling-free period of at least 1 month's duration, familial and social support and preferred type of gambling and medium for gambling. We used the Latent GOLD software ${ }^{43} 44$ to perform a latent class clustering (LCC) of the gamblers.
LCC is a person-centred approach that seeks to identify homogeneous subgroups, each group being defined by a specific probability distribution. LCC has been found to be more likely to give superior classification results than more traditional methods such as $k$-means or hierarchical clustering, ${ }^{45}$ particularly because it requires fewer assumptions. Moreover, LCC can handle mixed-mode data (ie, both categorical and continuous variables) without transformation of variables. We ran models that comprised one to six clusters. Missing data were supposed missing at random because these missing data were considered independent of the values of the variable but dependent on another variable (eg, missing values for age at onset of gambling problems were independent of the variable of age at onset of gambling problems, and depended instead on whether or not the participant had ever had gambling problems at all). Missing data were handled with full information maximum likelihood (FIML) estimation. FIML consists of estimating a likelihood function for each individual based on the data that are not missing so that all the available data are used. The Bayesian information criterion (BIC) (a lower BIC indicating a better model) and the classification error rate (which represents the precision of individuals' classification) were used to select the best model. Statistical differences between clusters were assessed by Wald tests. Variables that do not influence the estimation of the model were included as covariates to describe and compare the clusters. For these inactive covariates, a three-step approach was used to test differences between clusters. This method provides an unbiased estimation of the association between clustermembership probabilities and external covariates by maximising a weighted log-likelihood function for clustered data. ${ }^{46}$

\section{RESULTS}

\section{Descriptive analysis}

The sample comprised 256 NPGs, 169 PGWTs and 203 PGSTs. The sociodemographic data of the whole sample $(\mathrm{n}=628)$ are detailed in table 3 . Participants were mainly men $(n=418,66.6 \%)$, and the mean age was 43.4 years $(S D=12.9)$. Most participants were employed, with a regular income higher than $€ 1100$ (ie, approximately 1400 US\$).

\section{Clustering}

Gambling characteristics were used for clustering: age at gambling initiation, age at problem gambling onset, duration of gambling history, age at diagnosis of first psychiatric comorbidities (if relevant, history of gambling-free period of at least 1 month, familial and social support related to gambling problems, preferred type of gambling, preferred medium of gambling). Among the six models tested, two were very similar in terms of the BIC indicator: a model with three clusters $(\mathrm{BIC}=18253.8)$ and another with four clusters $(\mathrm{BIC}=18252.6)$. Of these two models, the one with three clusters displayed a lower classification 
Table 3 Descriptive analysis of sociodemographic variables for the whole sample

\begin{tabular}{|c|c|c|}
\hline & $N$ & $\%$ \\
\hline \multicolumn{3}{|l|}{ Gender } \\
\hline Male & 418 & 66.6 \\
\hline Female & 210 & 33.4 \\
\hline \multicolumn{3}{|l|}{ Marital status } \\
\hline Single & 313 & 49.9 \\
\hline In a relationship & 314 & 50.1 \\
\hline \multicolumn{3}{|l|}{ Educational level } \\
\hline Below high school graduation & 306 & 48.9 \\
\hline $\begin{array}{l}\text { Higher than or equal to high school } \\
\text { graduation }\end{array}$ & 320 & 51.1 \\
\hline \multicolumn{3}{|l|}{ Professional activity } \\
\hline Working & 398 & 63.5 \\
\hline Not working & 229 & 36.5 \\
\hline \multicolumn{3}{|l|}{ Level of income } \\
\hline $\begin{array}{l}\text { Regular and higher than the French } \\
\text { minimum wage (approximately } € 1100 \\
\text { or } 1400 \text { US\$) }\end{array}$ & 440 & 70.1 \\
\hline \multirow[t]{2}{*}{ Others } & 188 & 29.9 \\
\hline & $M$ & $S D$ \\
\hline Age & 43.4 & 12.9 \\
\hline
\end{tabular}

error rate $(7.92 \%$ vs $13.80 \%$ for the four-cluster solution) and was better suited to clinical interpretation. We thus chose the three-cluster partition.

Clinical, socio-professional, psychological variables and gambling characteristics of the three clusters are presented in table 4.

We identified three clusters with significant differences among the courses of gambling. Figure 1 represents the three clusters and the significant variables and covariables.

\section{The early onset and short course cluster (47.5\%)}

This group was predominantly represented by young men $(76.5 \%$ men, mean age 32.7$)$. This group was more active than the others $(73.0 \%$ were active), and more than half were single $(55.4 \%)$. This cluster has the lowest onset age (16.4 years old) and the earliest beginning of problem gambling (26.5 years old). Problem gambling thus appeared approximately 10 years after gambling initiation. Moreover, psychiatric comorbidities appeared earlier in life (15.3 years). Nearly half of this cluster (46.1\%) had a history of mood disorders, with the highest frequency of lifetime hypomanic or manic episodes $(13.6 \%)$. This cluster had significantly the highest level of Novelty Seeking $(M=54.9)$. Addictive comorbidities were also more prevalent in this group, with $28.7 \%$ and $25.5 \%$, respectively, reporting an alcohol use disorder and a substance use disorder. This group was the only one with a majority of PG (52\%) and had the highest proportion of PGST (35.2\%). Seventy-one per cent had already experienced at least 1 month without gambling, which was significantly higher than in other groups. For the two other clusters, the favourite type of gambling was predominantly pure chance games $(48.2 \%)$. However, contrariwise, a majority of these gamblers identified strategic games (regrouping bank games with an element of skill and social games with skill) as their preferred gambling type $(51.8 \%)$. Moreover, $20 \%$ preferred gambling on the Internet, a higher proportion than in the other clusters.

\section{The early onset and long course cluster (35.0\%)}

This group was also predominantly constituted by men (67.6\%), but was older than in the EarlyOnset and Short Course (EOSC) cluster ( $M=53.4$ years old). The majority lived with a partner $(61.3 \%)$, contrary to the two other clusters, even though they were those who reported the lowest frequency of familial support $(84.3 \%)$. The mean gambling onset age was 17.9 years. Problem gambling in problem gamblers appeared 23.4 years after gambling initiation, and the mean duration of the gambling practice at the moment of the inclusion was 35.4 years, which was more than twice the length for the two other clusters. Mooddisorders were present in $45.5 \%$ and anxietydisorders in $36.4 \%$. Alcohol use disorders were quite prevalent in this cluster (27.0\%). Psychiatric comorbidities appeared late in life, with the mean age of the first diagnosis of psychiatric comorbidity being 23.7 years, much older than the mean age of gambling initiation. They had the highest prevalence of suicidal attempts (18.2\%) and a higher frequency of current suicidal risk than the EOSC cluster $(26.7 \%)$. They had the lowest prevalence of gamblers having experienced a gambling cessation for at least 1 month $(53.6 \%)$. The majority $(51.6 \%)$ of this cluster was NPG, and the proportion of PGWT was the highest in this group (23.0\%). This group had the highest proportion of bank games with an element of skill as the preferred gambling type (36.1\%). Slightly less than $10 \%$ preferred Internet gambling.

\section{The late onset and short course cluster (17.5\%)}

This group was the only one predominantly constituted by women $(62.5 \%)$, with a mean age of 52.3 years old. However, the majority lived alone $(57.0 \%)$, and this cluster had the lowest prevalence of active employment $(52.0 \%)$. The mean age of gambling initiation was 36.1 years old, significantly older than the other two groups. However, the gambling course between the mean age of initiation and the mean age of problem gambling was the shortest among the whole sample: 9 years. Psychiatric comorbidities were more prevalent in this group; $56.0 \%$ had a history of mood disorders, especially a high prevalence of lifetime depressive disorder $(48.6 \%)$. Currentsuicidal risk was reported in $29.5 \%$, more than the two other groups. Psychiatric comorbidities appeared later in life. Indeed, the mean age of the first diagnosis of psychiatric comorbidity was 26.5 years old. The frequency of addictive disorders was the lowest in this group (19.8\%). They 
Table 4 Profiles of the three clusters identified

\begin{tabular}{|c|c|c|c|c|}
\hline & Cluster 1 & Cluster 2 & Cluster 3 & $P$ value \\
\hline & EOSC & EOLC & LOSC & Wald test \\
\hline Cluster size \%, $\mathrm{N}$ & $47.5(298)$ & $35.0(220)$ & $17.5(110)$ & \\
\hline \multicolumn{5}{|l|}{ Variables included in the clustering analysis } \\
\hline Age at gambling initiation (yrs) & 16.4 & 17.9 & 36.1 & $<0.001^{\star} \dagger \ddagger$ \\
\hline Age at onset of problem gambling (yrs) & 26.5 & 41.3 & 45.1 & $<0.001^{\star} \dagger \ddagger$ \\
\hline Duration of gambling history (yrs) & 16.2 & 35.4 & 16.2 & $<0.001^{\star} \ddagger$ \\
\hline Age at diagnosis of the first psychiatric comorbidities (if relevant) (yrs) & 15.3 & 23.7 & 26.5 & $<0.001^{*} \dagger$ \\
\hline History of gambling-free period of at least 1 month (\%) & 71.0 & 53.6 & 58.3 & $0.001^{\star} \ddagger$ \\
\hline Familial and social support related to gambling problems (\%) & 92.6 & 84.3 & 92.5 & $0.018^{*}$ \\
\hline \multicolumn{5}{|l|}{ Preferred type of gambling } \\
\hline $\begin{array}{l}\text { Pure chance games (lotteries, slots, scratch cards, video lottery } \\
\text { terminals, etc) }\end{array}$ & 48.2 & 58.2 & 79.8 & $<0.001^{\star} \ddagger$ \\
\hline $\begin{array}{l}\text { Bank games with an element of skill (sports betting, horse race } \\
\text { betting, blackjack) }\end{array}$ & 30.8 & 36.1 & 16.9 & \\
\hline Social games with skill (Texas hold'em and Omaha variants of poker) & 21.0 & 5.7 & 3.4 & \\
\hline \multirow[t]{2}{*}{ Preferred gambling medium (on the Internet) } & 20.0 & 8.1 & 2.3 & $<0.001^{*} \dagger$ \\
\hline & & & & $<0.001^{*} \dagger$ \\
\hline \multicolumn{5}{|l|}{ Covariables (not included in clustering) } \\
\hline \multicolumn{5}{|l|}{ Sociodemographic characteristics } \\
\hline \multicolumn{5}{|l|}{ Gender (\%) } \\
\hline Women & 23.5 & 32.4 & 62.5 & $<0.001^{*} \dagger \ddagger$ \\
\hline Men & 76.5 & 67.6 & 37.5 & \\
\hline Age (yrs) & 32.7 & 53.4 & 52.3 & $<0.001^{*} \dagger$ \\
\hline Level of income $\geq € 1100$ (1400 US\$) (\%) & 67.2 & 72.8 & 72.4 & 0.250 \\
\hline Marital status (single) (\%) & 55.4 & 38.7 & 57 & $<0.001^{\star} \ddagger$ \\
\hline Employment status (active) (\%) & 73.0 & 56.0 & 52.0 & $<0.001^{*} \dagger$ \\
\hline \multicolumn{5}{|l|}{ Gambling severity } \\
\hline \multicolumn{5}{|l|}{ Gambling status (\%) } \\
\hline Non-problem gamblers & 48.1 & 51.6 & 55.7 & $0.020^{*} \ddagger$ \\
\hline Problem gamblers without treatment & 16.8 & 23.0 & 21.3 & \\
\hline Problem gamblers seeking treatment & 35.2 & 25.4 & 23.0 & 0.200 \\
\hline Number of DSM-IV criteria (mean) & 3.8 & 3.7 & 3.3 & 0.200 \\
\hline \multicolumn{5}{|l|}{ Gambling habits: } \\
\hline Amount wagered monthly in gambling (in euros) & & & & 0.560 \\
\hline Gambling frequency over the past 12 months (\%) & & & & $0.067 \ddagger$ \\
\hline Less than once a month & 8.2 & 6.9 & 8.6 & \\
\hline More than once a month & 17.6 & 12.9 & 19.2 & \\
\hline Once a week & 13.2 & 14.6 & 20.6 & \\
\hline More than once a week & 61.0 & 65.7 & 51.6 & \\
\hline \multicolumn{5}{|l|}{ Comorbid psychiatric disorders } \\
\hline Mood disorders (\%) & 46.1 & 45.5 & 56.0 & 0.110 \\
\hline Depressive episode (\%) & 40.2 & 41.7 & 48.6 & 0.260 \\
\hline Hypomanic or manic episode (\%) & 13.6 & 9.2 & 12.9 & 0.240 \\
\hline Anxiety disorders (\%) & 38.2 & 36.4 & 40.0 & 0.780 \\
\hline Panic disorder (with or without agoraphobia) & 18.4 & 21.4 & 25.4 & 0.220 \\
\hline
\end{tabular}




\begin{tabular}{|c|c|c|c|c|}
\hline & Cluster 1 & Cluster 2 & Cluster 3 & $P$ value \\
\hline & EOSC & EOLC & LOSC & Wald test \\
\hline Social phobia & 11.7 & 8.4 & 15.9 & 0.082 \\
\hline Generalised anxiety disorder & 17.4 & 12 & 11.7 & 0.08 \\
\hline Post-traumatic stress disorder & 7.2 & 6.3 & 9.7 & 0.490 \\
\hline Alcohol use disorder & 28.7 & 27.0 & 13.7 & 0.007 \\
\hline Substance use disorder & 25.5 & 10.1 & 6.1 & $<0.001$ \\
\hline Antisocial personality disorder (\%) & 5.5 & 3.3 & 1.1 & 0.220 \\
\hline Current suicide risk (\%) & 19.4 & 26.7 & 29.5 & $0.027^{*} \dagger$ \\
\hline Personality & 54.9 & & & \\
\hline \multicolumn{5}{|l|}{ TCl scores (M) } \\
\hline Novelty seeking & 54.9 & 51.5 & 48.6 & $0.001^{*} \dagger$ \\
\hline Harm avoidance & 43.7 & 44.7 & 42.8 & 0.730 \\
\hline Determination & 67.8 & 67.0 & 68.7 & 0.720 \\
\hline Cooperation & 73.6 & 73.5 & 75.6 & 0.450 \\
\hline Reward dependence & 60.8 & 59.1 & 60.7 & 0.460 \\
\hline Transcendence & 27.7 & 33.8 & 36.7 & $<0.001^{*} \dagger$ \\
\hline Persistence & 52.8 & 55.9 & 59.2 & $0.069 \dagger$ \\
\hline
\end{tabular}

*Significant test $(\mathrm{p}<0.05)$ for the comparison between cluster 1 and cluster 2. †Significant test $(p<0.05)$ for the comparison between cluster 1 and cluster 3 . $\neq$ Significant test $(p<0.05)$ for the comparison between cluster 2 and cluster 3.

ADHD, attention deficit/hyperactivity disorder; DSM-IV, Diagnostic and Statistical Manual of Mental Disorders, Fourth Edition; EOLC, Early Onset and Long Course; EOSC, Early Onset and Short Course; LOSC, Late Onset and Short Course; TCI, temperament and character inventory; WURS, Wender-Utah Rating Scale; yrs, years.

displayed significantly the highest personality scores for transcendence $(M=36.7)$. In this cluster, the frequency of NPG was the highest $(55.7 \%)$, and the problem gamblers were equally either seeking treatment $(23.0 \%)$ or not $(21.3 \%)$. The preferred type of gambling was predominantly pure chance games for $79.8 \%$ of the cluster, and only $2.3 \%$ preferred Internet gambling.

\section{DISCUSSION}

\section{Main findings}

Three different profiles of gamblers

Three distinct clinical profiles of gamblers were identified through the analysis of gambling characteristics. Differences in the course and characteristics of gambling corresponded to psychopathological profiles, and they were significant in terms of sociodemographic variables (gender, age, work and marital status) and also in terms of gambling status and some comorbidities (addictive disorders, hypomanic episodes and personality traits). We propose a classification of our clusters according to gambling characteristics: Early Onset and Short Course (EOSC) (Cluster 1), Early Onset and Long Course (EOLC) (Cluster 2) and Late Onset and Short Course (LOSC) (Cluster 3). Regarding significant clinical and psychopathological covariables, this three-cluster model defined through the analysis of gambling characteristics is partly comparable to the theoretical pathways model of Blaszczynski and Nower, ${ }^{17}$ which was in turn defined through psychopathological variables.

\section{The early onset and short course cluster (cluster 1)}

The mean age at gambling initiation is under 18 years, which is the legal age for gambling in France. Lynch et al found that young adults who had started gambling prior to the age of 18 were more likely to experience at least one symptom of GD than those who started gambling at the age of 18 or later. ${ }^{47}$ Early initiation is described as a marker of risk of GD in later life: Kessler et al (2008) found that the mean age at the onset of gambling was significantly lower among those who subsequently developed problem gambling than among those who did 
Figure 1. Clustering : 3 clusters and significant variables and covariables

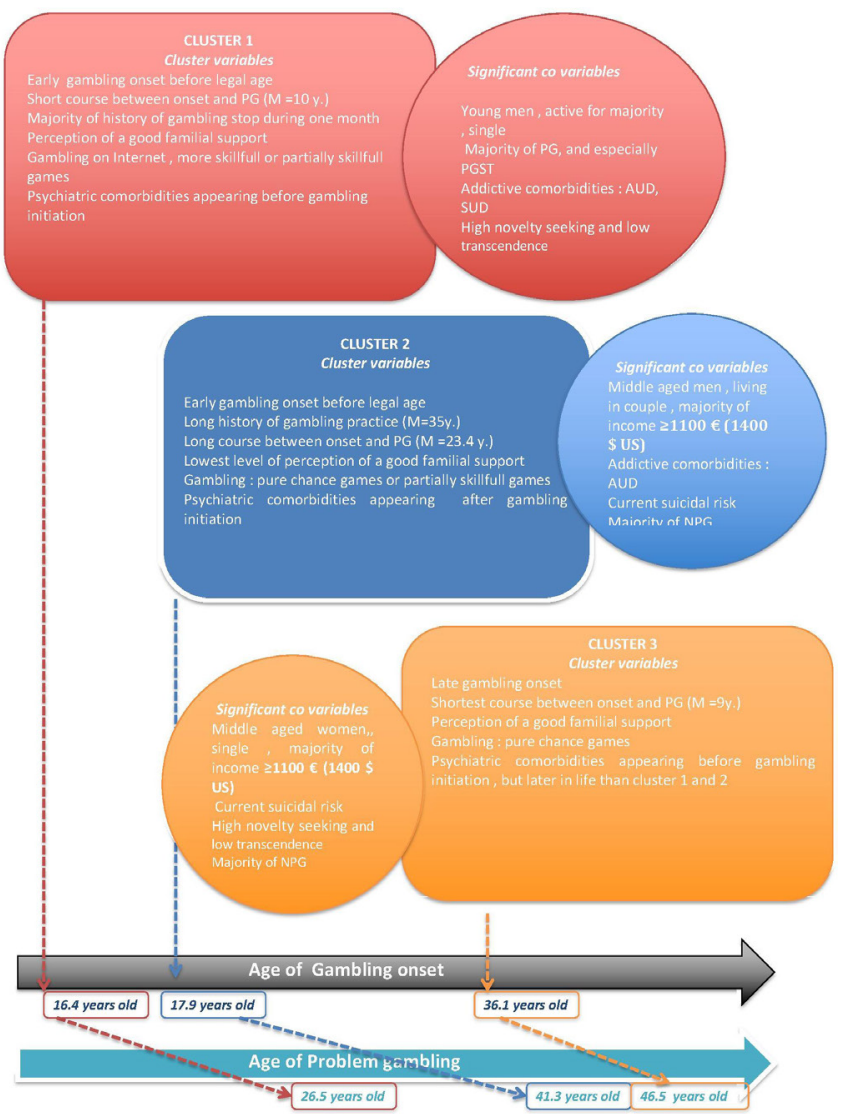

Figure 1 Clustering: three clusters and significant variables and covariables. AUD, alcohol use disorder; NPG, nonproblem gamblers; PG, problem gamblers; PGST,problem gamblers seeking treatment; SUD, substance use disorder.

not, ${ }^{48}$ and Jiménez-Murcia et al identified a younger age at the onset of gambling as associated with a greater severity of problem gambling. Our results agreed with this hypothesis: the EOSC group had a higher frequency of problem gambling and higher gambling severity than the two other groups. Nevertheless, age at initiation did not explain the course of gambling in itself: the two clusters associated with the earlier age at onset presented two different courses of gambling, either a short development (approximately 10 years) for the EOSC cluster or a long development (approximately 23 years) for the EOLC cluster.

This cluster had a significantly higher preference for online gambling than the others. Gambling on the Internet is associated with a high availability of gambling opportunity, and online poker induces specific problems, leading problem gamblers to lose money but also to spend a large amount of time gambling. ${ }^{50}$ Moreover, involvement in gambling and attraction to strategic games are risk factors for problem gambling. ${ }^{51}$ Male Internet bettors face heightened risks of related gambling problems. ${ }^{52}$ Mirroring the comments made above regarding EGM players, much may depend on the patterns of use exhibited by online sports betters. For example, if online betting is done sporadically in a social context (eg, watching a game together), then online play may represent no extra risk over venue-based play. On the other hand, if online sports betting facilitates different patterns of use (eg, solitary betting in extended sessions late at night), then this would provide further evidence that the online product presents a greater risk. At the present time, concern appears to be justified, as young men in particular are increasingly seeking treatment for difficulties in controlling their online sports betting. ${ }^{53}$

Strategic games usually attract young males, with an earlier onset of the disorder. ${ }^{54}$ They tend to engage in more than one specific type of problematic game and to take more risks, including betting large amounts of money. ${ }^{54}$ This cluster included the highest frequency of PG and the highest frequency of PGST, and they most frequently indicated the occurrence of gambling-free periods of 1 month, probably in an effort to regain control over gambling or in reaction to negative consequences.

The EOSC cluster presented the highest level of ADHD symptomatology. One may suppose that psychopathological variables combined with age at onset may influence the course of gambling, as defined in the pathways model. ${ }^{17}$ Impulsivity is also a key point of the antisocial impulsivist pathway, defined by Blaszczynski and Nower as being associated with gambling-related criminal behaviours and addictive comorbidities. ${ }^{17}$ Novelty Seeking was also high in our study, as reported in the literature, especially in social games with skill. ${ }^{54}$ This was associated with a high prevalence of psychiatric and addictive comorbidities, as previously described for PG with impulsivity and ADHD. ${ }^{175657}$

\section{EOLC cluster (cluster 2)}

The EOLC cluster had a long course of gambling (35.4 years). They spent the highest amount of money on gambling, and only $53.6 \%$ had experienced a gambling cessation for at least 1 month.

We found important results concerning familial support: although EOLC gamblers lived predominantly with partners, they reported the lowest level of familial and social support related to gambling problems. We hypothesise that gambling had severe consequences on the family, as shown in the literature, ${ }^{58}$ thus reducing familial support.

An important characteristic is the absence of any lifetime premorbid feature of psychopathology before the onset of the gambling problem. We may hypothesise that symptoms of anxiety or depression were the consequences of problem-gambling-related difficulties. However, at inclusion, they presented the highest prevalence of attempted suicide (and a current suicide risk in $26.7 \%$ of cases). One can assume suicide risk and attempted suicides to be consequences of problem-gambling-related difficulties. ${ }^{60}$ The EOLC cluster could share similarities with the first pathway, defined by Blaszczynski and Nower as 'conditioned problem gamblers' (CG). This CG group is defined as gamblers without comorbidities who are 
preoccupied with gambling, engage in chasing and fluctuate between excessive gambling and problem gambling.

\section{The LOSC cluster (cluster 3)}

The LOSC gamblers predominantly preferred pure chance games. Different studies have shown that gamblers who preferred slot machines had higher levels of depression. $^{2561}$

We can underline two notable points in our LOSC cluster. First, women made up the majority of the LOSC cluster $(62.5 \%)$, and in this cluster the course of gambling was the shortest observed in our study. These results support the concept of a 'telescoping effect' in the course of problem gambling among women. ${ }^{62-64}$ In the literature, this effect was indicated by female gamblers seeking treatment, ${ }^{6566}$ but was not confirmed in the general population. ${ }^{67}$ In our study, we included a mixed population of gamblers (NPG, PGWT and PGST), and our sample also consisted of gamblers who were somewhat older $(M=52$ years in the LOSC cluster) than in the Slutske sample. ${ }^{67}$

Second, the LOSC gamblers had the highest mean age at gambling initiation, but also the shortest gambling trajectory. As a consequence, we could conclude that the course of gambling and the risk of problem gambling were not systematically linked to an early age of onset, but perhaps to a combination of psychopathological variables, age at initiation, gender and type of gambling, as hypothesised in some studies. ${ }^{20} 21$

This cluster presented premorbid anxiety and/or depression: $56.0 \%$ had a lifetime history of mood disorder, with $29.5 \%$ experiencing a current suicide risk, which are the highest percentages of our sample. This cluster was also notable for the lowest frequency of seeking treatment. Suicide risk is one of the main dramatic consequences of gambling, ${ }^{60}$ and suicidal thoughts can lead to giving up gambling and accepting treatment. This course of gambling characteristic could support the hypothesis that participation in gambling is motivated by desire on the part of gamblers to modulate their emotional distress and their negative feelings. ${ }^{17}$ The LOSC cluster might share similarities with the pathway of 'emotionally vulnerable problem gamblers'. ${ }^{1727} 28$

\section{Strengths and weaknesses of this study}

This study has several limitations, especially the relatively restricted amount of data collected. Characteristics related to the course of gambling (age at initiation, age at onset of gambling problems, etc) and lifetime psychiatric disorders were assessed in a retrospective manner and were self-reported, which could induce a recall bias. Moreover, we did not use DSM-5 criteria, and we did not analyse impulsivity and cognitive distortions in this population. Recruitment of patients occurred at the moment of the legalisation of online gambling in France (2010), which limits generalisation, as online gambling has since become widely available. However, the combination of NPGs and PGs who have and who have not sought treatment is one of the main strengths of our study. We also included gamblers recruited directly from gambling locations. This method gave us access to a broad spectrum of gambling activities. Finally, this sample size has rarely been achieved by studies with semi-structured interviews (studies with such high numbers of participants are generally based on telephone-based surveys).

That study more accurately categorised profiles of gamblers and problem gamblers through gambling characteristics and course. It did not examine factors that increase the risk of transitioning from a non-problem gambler to a problem gambler, nor did it define vulnerability profiles associated with the emergence of problem gambling in a longitudinal study.

\section{Implications for clinicians and policymakers}

It is important to consider gambling characteristics because preventive interventions or policy measures may reduce the risk of problem gambling or minimise harm from gambling. ${ }^{7}$ We defined a clustering of gamblers through the analysis of variables which were easy to identify by psychiatrists or physicians in primary care: gambling characteristics, age at gambling initiation, type of gambling and gambling medium, duration of gambling activities, age at onset of psychiatric comorbidities and history of gambling-free periods of at least 1 month's duration. We hypothesise that simple screening concerning these gambling characteristics could be constructed to prevent PG and to help PG identification.

Prevention interventions should be tailored to these at-risk profiles. The preceding results imply that interventions need to particularly target prevention at different profiles: young adult males gambling online in strategic games, for example, or middle-aged women gambling offline to hazardous games. Public health messages should be available online and offline which are adapted to these profiles. Clinical programmes could also be adapted to these specific profiles, for we may suppose that with respect to the respective cognitive distortions, motivational training would certainly differ between these profiles.

The gambling characteristics identified in this study as significantly different between the three clusters are easily identified but could also be target of specific intervention to reduce harm in gambling, especially in at-risk profiles.

We demonstrated that a large majority of gamblers in this sample started to gamble before the legal age of 18 years. For gambling prevention policy, it is worth noting that despite the ban on gambling under 18 years of age, minors can gamble very early. We stress the need to develop early interventions to provide information and prevent gambling, especially among adolescents. Prevention programmes could include targeted interventions for youth to explain gambling risks and how to avoid them. One explanation for the framing of risky gambling behaviour of youth may be gambling advertising and availability. Gambling marketing is present in many media (advertising posters, the Internet, magazines, television, etc) that are accessible to adolescents, and its content 
is very attractive. A recent study showed that marketing significantly influences attitudes towards gambling, gambling behaviours and intention to participate. ${ }^{68}$

We also should pay attention to the type of gambling and the medium used for gambling. In our study, strategic games and Internet gambling seemed to be associated with more serious gambling profiles and short courses of gambling development in younger men. Targeted prevention on the Internet, especially regarding strategic games, could be developed to reduce harm from gambling. Contrariwise, a large majority of the LOSC cluster preferred to gamble in pure chance games offline. Unlike casino or Internet games, pure chance offline games (especially scratch games) in France do not include any self-exclusion programmes, and identity controls are not systematic when a ban is in place. Consequently, French gamblers with gambling problems participating in pure chance games offline are less able to put in place safeguards that may help them to quit or reduce gambling. This point is a public health concern, particularly when we consider, as in our study, that gambling problems occur in vulnerable persons. These results raise questions about the need for governments to develop policies and regulations to reduce young people's exposure to gambling products and marketing and to protect vulnerable persons from harm caused by gambling.

\section{Author affiliations}

${ }^{1}$ EA 7479 SPURB0, Universite de Bretagne Occidentale, Brest, France

${ }^{2}$ Addictive Disorders Department, CHRU de Brest, Brest, France

${ }^{3}$ UMR 1246 SPHERE, Université de Nantes, Nantes, France

${ }^{4}$ Addictology and Psychiatry Department, CHU Clermont Ferrand, Clermont Ferrand, France

${ }^{5}$ EA 4430 CLIPSYD 'Clinique psychiatrique developpement', Universite ParisNanterre, Nanterre, France

${ }^{6}$ Addictive Disorders, Hospital Louis-Mourier, Colombes, France

${ }^{7}$ Addictive disorders Unit Marmottan, GPS Perray-Vaucluse, Épinay-sur-Orge, France ${ }^{8}$ Department of Adult Psychiatry, Hopital Sainte-Marguerite, Marseille, France

${ }^{9}$ Psychiatric Laboratory SANPSY USR 3413, University of Bordeaux, Talence, France

${ }^{10}$ Addictive Disorders, Hospital Centre Charles Perrens, Bordeaux, France

${ }^{11}$ Psychiatry and Addictology Department, Hôpital Paul Brousse, APHP Villejuif,

Villejuif cedex, France

${ }^{12}$ CESP, Université Paris-Saclay, Univ. Paris-Sud, UVSQ, CESP, INSERM, Villejuif,

France

${ }^{13}$ CMAP, Ecole Polytechnique, Palaiseau Cedex, France

${ }^{14}$ Psychiatry and Addictology, CHU Clermont Ferrand, Clermont Ferrand, UK

${ }^{15}$ Addictology, University Hospital of Nantes, Nantes, France

${ }^{16}$ Addictology and Psychiatry Department, University Hospital of Nantes, Nantes, France

Acknowledgements We wish to sincerely thank all the staff who contributed to this study (the JEU Group) for their valuable assistance and significant investment. Special thanks goes to those who collected the data. The members of the JEU Group are: Marie Grall-Bronnec, Gaëlle Challet-Bouju, Jean-Luc Vénisse, Lucia Romo, Cindy Legauffre, Caroline Dubertret, Irène Codina, Marc Valleur, Marc Auriacombe, Mélina Fatséas, Jean-Marc Alexandre, Pierre-Michel Llorca, Isabelle Chéreau-Boudet, Christophe Lançon, David Magalon, Michel Reynaud and Amandine Luquiens. This research was conducted at the initiative of and coordinated by the Addictology and Psychiatry Department of the University Hospital of Nantes, who sponsored this study.

Collaborators JEU Group: Marie Grall-Bronnec, Gaëlle Challet-Bouju, Jean-Luc Vénisse, Lucia Romo, Cindy Legauffre, Caroline Dubertret, Irène Codina, Marc Valleur, Marc Auriacombe, Mélina Fatséas, Jean-Marc Alexandre, Pierre-Michel
Llorca, Isabelle Chéreau-Boudet, Christophe Lançon, David Magalon, Michel Reynaud, Amandine Luquiens.

Contributors MGB and GCB designed the JEU cohort study, conducted the literature search and wrote the protocol. MGB and GCB were responsible for project management. BP provided methodological advice, designed the statistical analysis plan and conducted the statistical analysis for this research. All authors (including those mentioned in the JEU Group) contributed to the recruitment and inclusion of the participants in the JEU cohort study and to the development of the protocol, especially to the selection of the assessment content. MGL, MGB, and GCB wrote the manuscript. All authors read and approved the final manuscript.

Funding The JEU cohort study was supported by joint support of the French Inter-Departmental Mission for the Fight against Drugs and Drug Addiction (MILDT) and the French National Institute of Health and Medical Research (INSERM), as part of the call for research projects launched by these two organisations in 2007 (MIL08010), and a grant from the French Ministry of Health (PHRC 2009 - RCB 2008-A01188-47). They had no role in the study design, collection, analysis or interpretation of the data, writing of the manuscript or decision to submit the paper for publication. The research presented here was conducted at the initiative of and coordinated by the Addictology and Psychiatry Department of Nantes University Hospital. Nantes University Hospital is the sponsor of this study. There were no constraints on publishing.

Competing interests MGB and GCB declare that the University Hospital of Nantes has received funding from the gambling industry (FDJ and PMU) in the form of a sponsorship that supports the gambling section of the BALANCED Unit (Reference Centre for Excessive Gambling). Scientific independence towards gambling industry operators is warranted. There were no constraints on publishing. LR declares that the University of Paris Ouest Nanterre La Défense has received funding directly from gambling industry (FDJ and PMU) as part of other research contracts - this funding has never had any influence on the present work.

Patient consent for publication Not required.

Ethics approval This study was approved by the French Research Ethics Committee (CPP) on 8 January 2009. The approval granted from the CPP applies to all sites at which the study occurred.

Provenance and peer review Not commissioned; externally peer reviewed.

Data availability statement № data are available.

Open access This is an open access article distributed in accordance with the Creative Commons Attribution Non Commercial (CC BY-NC 4.0) license, which permits others to distribute, remix, adapt, build upon this work non-commercially, and license their derivative works on different terms, provided the original work is properly cited, appropriate credit is given, any changes made indicated, and the use is non-commercial. See: http://creativecommons.org/licenses/by-nc/4.0/.

ORCID iDs

Morgane Guillou Landreat http://orcid.org/0000-0003-1568-9480

Amandine Luquiens http://orcid.org/0000-0002-9402-442X

\section{REFERENCES}

1 Calado F, Griffiths MD. Problem gambling worldwide: an update and systematic review of empirical research (2000-2015). J Behav Addict 2016;5:592-613.

2 Costes J-M, Kairouz S, Eroukmanoff V, et al. Gambling patterns and problems of gamblers on licensed and unlicensed sites in France. $J$ Gambl Stud 2016;32:79-91.

3 Shaffer HJ, Korn DA. Gambling and related mental disorders: a public health analysis. Annu Rev Public Health 2002;23:171-212.

4 Williams RJ, Volberg RA, Stevens RMG. The population prevalence of problem gambling: methodological influences, standardized rates, jurisdictional differences, and worldwide trends. Report prepared for the Ontario Problem Gambling Research Centre and the ntario Ministry of Health and Long term Care; 2012.

5 The Lancet. Problem gambling is a public health concern. The Lancet 2017;390:913.

6 Hing N, Cherney L, Gainsbury SM, et al. Maintaining and losing control during internet gambling: A qualitative study of gamblers' experiences. New Media Soc 2015;17:1075-95.

7 Hing N, Russell AM, Browne M. Risk factors for gambling problems on online electronic gaming machines, race betting and sports betting. Front Psychol 2017;8:779. 
8 De Luigi N, Gibertoni D, Randon E, et al. Patterns of Gambling Activities and Gambling Problems Among Italian High School Students: Results from a Latent Class Analysis. J Gambl Stud 2018;34:339-59.

9 American Psychiatric Association. Diagnostic and statistical manual of mental disorders. 5th ed. Washington: DC, 2013.

10 Stucki S, Rihs-Middel M. Prevalence of adult problem and pathological gambling between 2000 and 2005: an update. J Gambl Stud 2007;23:245-57.

11 Bruneau M, Grall-Bronnec M, Vénisse J-L, et al. Gambling transitions among adult gamblers: a multi-state model using a Markovian approach applied to the JEU cohort. Addict Behav 2016;57:13-20.

12 Wardle Het al. The British Gambling prevalence survey 2010. London: The Stationery Office, 2011.

13 Costes JM. Levels and gambling practices in 2010 Lettre tendances; 2011: 1-8.

14 Abbott MW, Romild U, Volberg RA. Gambling and problem gambling in Sweden: changes between 1998 and 2009. J Gamb/ Stud 2014;30:985-99.

15 Guillou Landreat M, Le Reste J-Y, Théréné-Mouden C, et al. [Gambling in elderly patients]. Soins Gerontol 2017;22:35-7.

16 Caillon J, Grall-Bronnec M, Bouju G, et al. [Pathological gambling in adolescence]. Arch Pediatr 2012;19:173-9.

17 Blaszczynski A, Nower L. A pathways model of problem and pathological gambling. Addiction 2002;97:487-99.

18 Valleur M, Codina I, Vénisse J-L, et al. Towards a validation of the three pathways model of pathological gambling. J Gamb/ Stud 2016;32:757-71.

19 Ledgerwood DM, Petry NM. Subtyping pathological gamblers based on impulsivity, depression, and anxiety. Psychol Addict Behav 2010;24:680-8.

20 Jiménez-Murcia S, Granero R, Tárrega S, et al. Mediational role of age of onset in gambling disorder, a path modeling analysis. J Gambl Stud 2016;32): :327-40.

21 Jiménez-Murcia S, Alvarez-Moya EM, Stinchfield R, et al. Age of onset in pathological gambling: clinical, therapeutic and personality correlates. J Gamb/ Stud 2010;26:235-48.

22 Burge AN, Pietrzak RH, Molina CA, et al. Age of gambling initiation and severity of gambling and health problems among older adult problem gamblers. PS 2004;55:1437-9.

23 Burge AN, Pietrzak RH, Petry NM. Pre/early adolescent onset of gambling and psychosocial problems in treatment-seeking pathological gamblers. J Gamb/ Stud 2006;22:263-74.

24 Gainsbury SM, Russell A, Blaszczynski A, et al. The interaction between gambling activities and modes of access: a comparison of Internet-only, land-based only, and mixed-mode gamblers. Addict Behav 2015:41:34-40.

25 Challet-Bouju G, Hardouin J-B, Renard N, et al. A gamblers clustering based on their favorite gambling activity. J Gamb/ Stud 2015;31:1767-88.

26 Dufour MC, Archer L, Gordis E. Alcohol and the elderly. Clin Geriatr Med 1992;8:127-42.

27 Moon M, Lister JJ, Milosevic A, et al. Subtyping Non-treatmentseeking problem gamblers using the pathways model. J Gamb/ Stud 2017;33:841-53.

28 Nower L, Blaszczynski A. Development and validation of the gambling pathways questionnaire (GPQ). Psychol Addict Behav 2017;31:95-109.

29 Suomi A, Dowling NA, Jackson AC. Problem gambling subtypes based on psychological distress, alcohol abuse and impulsivity. Addict Behav 2014;39:1741-5.

30 Nower L, Martins SS, Lin K-H, et al. Subtypes of disordered gamblers: results from the National epidemiologic survey on alcohol and related conditions. Addiction 2013;108:789-98.

31 Mallorquí-Bagué N, Tolosa-Sola I, Fernández-Aranda F, et al. Cognitive deficits in executive functions and decision-making impairments cluster gambling disorder sub-types. J Gamb/ Stud 2018;34:209-23.

32 Challet-Bouju G, Hardouin J-B, Vénisse J-L, et al. Study protocol: the JEU cohort study--transversal multiaxial evaluation and 5year follow-up of a cohort of French gamblers. BMC Psychiatry 2014; $14: 226$.

33 Toce-Gerstein M, Gerstein DR, Volberg RA. A hierarchy of gambling disorders in the community. Addiction 2003;98:1661-72.

34 Potenza MN. Should addictive disorders include non-substancerelated conditions? Addiction 2006;101 Suppl 1:142-51.

35 Toneatto T, Millar G. Assessing and treating problem gambling: empirical status and promising trends. Can J Psychiatry 2004;49:517-25.

36 Boutin C. " Le jeu: chance ou stratégie. In: I'homme L.é.d., ed. Choisir librement la place du jeu dansvotre vie. Quebec, 2010.
37 Lecrubier Y, Sheehan DV, Weiller E, et al. The mini international neuropsychiatric interview (mini). A short diagnostic structured interview: reliability and validity according to the CIDI. European Psychiatry 1997:12:224-31.

38 Ward MF, Wender PH, Reimherr FW. The Wender Utah rating scale: an aid in the retrospective diagnosis of childhood attention deficit hyperactivity disorder. Am J Psychiatry 1993;150:885-90.

39 Caci HM, Bouchez J, Baylé FJ. An aid for diagnosing attentiondeficit/hyperactivity disorder at adulthood: psychometric properties of the French versions of two Wender Utah rating scales (WURS-25 and WURS-K). Compr Psychiatry 2010;51:325-31.

40 De Fruyt F, Van De Wiele L, Van Heeringen C. Cloninger's Psychobiological model of temperament and character and the FiveFactor model of personality. Pers Individ Dif 2000;29:441-52.

41 Cloninger CR, Svrakic DM, Przybeck TR. A psychobiological mode of temperament and character. Arch Gen Psychiatry 1993;50:975-90.

42 Chakroun N, Faytout M, Pelissolo A, et al. French validation of a short version of the temperament and character inventory $(\mathrm{TCl}-125)$. Journal de Thérapie Comportementale et Cognitive 2005;15:27-33.

43 Vermunt JK, Magidson JHagenaars JA, McCutcheon AL, eds. Latent class cluster analysis in applied latent class analysis C.U. Cambridge p, 2002: 89-106.

44 Vermunt JK, Magidson J. Upgrade Manual for Latent GOLD 5.1 Belmont Massachusetts: Statistical Innovations Inc. In: Statistical innovations, 2016

45 Magidson J, Vermunt JK. Latent class models for clustering: a comparison with k-means. Canadian Journal of Marketing Research 2002;20:36-43.

46 Vermunt JK. Latent class modeling with covariates: two improved three-step approaches. Political Analysis 2010;18:450-69.

47 Lynch WJ, Maciejewski PK, Potenza MN. Psychiatric correlates of gambling in adolescents and young adults grouped by age at gambling onset. Arch Gen Psychiatry 2004;61:1116-22.

48 Kessler RC, Hwang I, LaBrie R, et al. Dsm-Iv pathological gambling in the National comorbidity survey replication. Psychol Med 2008:38:1351-60.

49 Slutske WS, Deutsch AR, Richmond-Rakerd LS, et al. Test of a potential causal influence of earlier age of gambling initiation on gambling involvement and disorder: a multilevel discordant twin design. Psychol Addict Behav 2014;28:1177-89.

50 Bonnaire C. [Internet gambling: what are the risks?]. Encephale 2012;38:42-9.

51 Mouneyrac A, Lemercier C, Le Floch V, et al. Cognitive characteristics of strategic and Non-strategic gamblers. J Gambl Stud 2018;34:199-208.

52 Milner Let al. An exploratory study of embedded gambling promotion in Australian football television broadcasts. Communication, Politics and Culture 2013;46:177-98.

53 Chantiri E. Online sports betting has created new generation of problem gamblers. Sydney: NSW, 2011

54 Moragas L, Granero R, Stinchfield R, et al. Comparative analysis of distinct phenotypes in gambling disorder based on gambling preferences. BMC Psychiatry 2015;15:86

55 Bonnaire C, Lejoyeux M, Dardennes R. Sensation seeking in a French population of pathological gamblers: comparison with regular and nongamblers. Psychol Rep 2004;94:1361-71.

56 Brandt L, Fischer G. Adult ADHD is associated with gambling severity and psychiatric comorbidity among treatment-seeking problem gamblers. J Atten Disord 2019;23:1383-1395.

57 Grall-Bronnec M, Wainstein L, Augy J, et al. Attention deficit hyperactivity disorder among pathological and at-risk gamblers seeking treatment: a hidden disorder. Eur Addict Res 2011;17:231-40.

58 Dowling NA, Rodda SN, Lubman DI, et al. The impacts of problem gambling on concerned significant others accessing web-based counselling. Addict Behav 2014;39:1253-7.

59 Chan EML, Dowling NA, Jackson AC, et al. Gambling related family coping and the impact of problem gambling on families in Hong Kong. Asian J of Gambling Issues and Public Health 2016;6:p. 1.

60 Guillou-Landreat M, Guilleux A, Sauvaget A, et al. Factors associated with suicidal risk among a French cohort of problem gamblers seeking treatment. Psychiatry Res 2016;240:11-18.

61 Bonnaire C, Bungener C, Varescon I. Subtypes of French pathological gamblers: comparison of sensation seeking, alexithymia and depression scores. J Gamb/ Stud 2009;25:455-71.

62 Bouju G, Grall-Bronnec M, Landreat-Guillou M, et al. Jeu pathologique : facteurs impliqués. L'Encéphale 2011;37:322-31.

63 Grall-Bronnec M, Wainstein L, Feuillet F, et al. Clinical profiles as a function of level and type of impulsivity in a sample group of at-risk and pathological gamblers seeking treatment. J Gamb/ Stud 2012;28:239-52. 
64 Zakiniaeiz Y, Cosgrove KP, Mazure CM, et al. Does Telescoping exist in male and female gamblers? does it matter? Front Psychol 2017;8:1510.

65 Grant JE, Odlaug BL, Mooney ME. Telescoping phenomenon in pathological gambling: association with gender and comorbidities. $J$ Nerv Ment Dis 2012;200:996-8.

66 Potenza MN, Steinberg MA, McLaughlin SD, et al. Gender-Related differences in the characteristics of problem gamblers using a gambling helpline. Am J Psychiatry 2001;158:1500-5.
67 Slutske WS, Piasecki TM, Deutsch AR, et al. Telescoping and gender differences in the time course of disordered gambling: evidence from a general population sample. Addiction 2015;110:144-51.

68 Pitt H, Thomas SL, Bestman A, et al. Factors that influence children's gambling attitudes and consumption intentions: lessons for gambling harm prevention research, policies and advocacy strategies. Harm Reduct J 2017;14:p. 11. 